\title{
Terapia cognitivo-comportamental para luto pela morte súbita de cônjuge
}

\section{Cognitive-behavioral therapy to mourning for the sudden death of spouse}

\author{
Adriana Cardoso de Oliveira e Silva ${ }^{1}$, Antonio Egidio Nardi ${ }^{2}$ \\ 1 Laboratório de Tanatologia e Psicometria da Universidade Federal Fluminense (UFF), INCT Translational Medicine, Programa de Pós-Graduação em Psiquiatria e Saúde Mental, Instituto de \\ Psiquiatria/UFRJ. \\ 2 Laboratório de Pânico e Respiração - Instituto de Psiquiatria/Universidade Federal do Rio de Janeiro (IPUB/UFRJ), INCT Translational Medicine, Instituto de Psiquiatria da Faculdade de Medicina, \\ Programa de Pós-Graduação em Psiquiatria e Saúde Mental da UFRJ.
}

\author{
Recebido: $16 / 11 / 2010$ - Aceito: 4/4/2011
}

\section{Resumo}

Introdução: $O$ processo de luto devido à morte súbita de cônjuge apresenta particularidades que devem ser consideradas no atendimento clínico daqueles que passam por essa experiência. Métodos: Estudo de caso de terapia para luto utilizando protocolo de atendimento cognitivista comportamental. O progresso da paciente foi avaliado no início e término do tratamento com Inventário Beck de Depressão (BDI), Inventário Beck de Ansiedade (BAI) e Escala Beck de Desesperança (BHS), Inventário de Sintomas de Stress de Lipp (ISSL) e Questionário de Saúde Geral (QSG). Resultados: Houve redução da depressão, ansiedade e desesperança. Em relação ao estresse, a paciente passou da fase de exaustão para a de resistência. Houve redução do estresse psíquico (de $95 \%$ para 50\%), da desconfiança em relação ao próprio desempenho (de $90 \%$ para $40 \%$ ), de distúrbios do sono (de $95 \%$ para $45 \%$ ), de desejo de morte (de $95 \%$ para $20 \%$ ) e também de distúrbios psicossomáticos (de 95\% para 60\%). Conclusão: O atendimento terapêutico para esse tipo específico de luto mediante o enfoque da terapia cognitivo-comportamental se mostrou eficaz.

Silva ACO, Nardi AE / Rev Psiq Clín. 2011;38(5):213-5

Palavras-chave: Luto, terapia cognitiva, terapia comportamental, tanatologia.

\begin{abstract}
Introduction: The mourning due to the sudden death of a spouse has special features that should be considered in therapy process of those who undergo this experience. Methods: Case work of grief therapy using a cognitive-behavioral protocol. The progress of the patient was assessed at the beginning and end of treatment with BDI, BAI, BHS, ISSL and GHQ. Results: There was reduction in depression, anxiety and hopelessness. Regarding stress, the patient, that was in the stage of exhaustion passed to resistance. Reduction of psychological stress (95\% to 50\%), distrust about their own performance (90\% to $40 \%$ ), sleep disturbances ( $95 \%$ to $45 \%$ ), death wish ( $95 \%$ to $20 \%$ ) and also of psychosomatic disorders (95\% to $60 \%$ ). Discussion: The therapeutic protocol through the focus of cognitive-behavioral therapy was effective for that specific type of mourning.
\end{abstract}

Silva ACO, Nardi AE / Rev Psiq Clín. 2011;38(5):213-5

Keywords: Bereavement, cognitive therapy, behavior therapy, thanatology.

\section{Introdução}

O luto é um quadro clínico que apresenta sinais e sintomas específicos ${ }^{1,2}$, tanto emocionais quanto cognitivos e comportamentais, diferentes dos quadros de depressão $0^{3,4}$. Os casos de luto decorrente de morte imprevista, assim como aqueles que envolvem a morte de um companheiro de longa data, apresentam particularidades ${ }^{5}$ que serão importantes na condução do planejamento terapêutico.

Enquanto a rede de apoio social, mais do que a qualidade do relacionamento com o cônjuge, exerce influência sobre a depressão e outros sintomas do luto, a qualidade dessa relação afeta a saudade percebida após a perda ${ }^{6}$. Entre jovens, é relatada maior incidência de depressão e alcoolismo nos dois anos seguintes a perdas por morte imprevista ${ }^{7}$.

No caso de morte de cônjuge, os parceiros sobreviventes sofrem grande pressão social para rapidamente retornarem às suas rotinas, tendo seus recursos internos fortemente mobilizados para conseguirem lidar com a tristeza e, simultaneamente, retomarem sua funcionalidade 8 . Pacientes em luto apresentam maior incidência de problemas psicossomáticos, busca por atendimento médico para queixas orgânicas e maior número de internações do que a população geral 9,10 .
Atualmente o luto é classificado no eixo $\mathrm{V}$, referente à avaliação global do funcionamento, do DSM-IV-TR, com código 62.82, e na categoria Z da CID-10. Estudos recentes avaliam a inclusão do luto entre os transtornos mentais no DSM-V e na CID-1111, mas, apesar disso, ainda são escassos os estudos disponíveis na literatura, voltados para a avaliação de procedimentos terapêuticos específicos para esse quadro.

Este estudo tem como objetivo apresentar os resultados obtidos no tratamento, com terapia cognitivo-comportamental, de um caso de luto devido à morte súbita de cônjuge.

\section{Relato do caso}

Paciente de 29 anos, feminina, com nível superior completo e emprego fixo, casada há três anos com homem de 37 anos, morto dois meses antes do primeiro atendimento psicológico. Na época em que o marido morreu, estavam planejando a gestação do primeiro filho. Moravam em residência própria e contavam com bom número de amigos e também com apoio familiar.

Ela conta que o marido era um homem saudável e que a morte ocorreu enquanto ele praticava atividade física ao ar livre. Segundo ela, "foi repentino, de repente o coração parou". Ele estava com amigos 
durante o ocorrido e foi levado ao hospital mais próximo do local, porém já estava morto quando chegou.

Durante o velório e o enterro, pôde contar com o apoio dos amigos e familiares, assim como nas duas semanas seguintes, porém, após esse período, relata que sentiu forte cobrança para retomar suas atividades, mesmo que ainda não se percebesse pronta para isso.

Solicitou dispensa do trabalho aproveitando férias que ela ainda não havia usufruído, depois disso, voltou a frequentar a instituição em que trabalhava, mas informa que "não conseguia produzir nada direito". Acrescenta que, por mais que tentasse dedicar-se às atividades do trabalho, sempre se "perdia pensando no que tinha acontecido".

Quando estava em casa, passava longas horas no quarto revendo fotos do casal ou olhando para os objetos que pertenciam ao marido. Não se desfez de nada do que era dele, permanecendo tudo "intocado desde a morte dele".

Relata que não conseguia dormir e passava a noite toda chorando. Quando, eventualmente, dormia, tinha "sonhos com a morte dele" e acordava sentindo-se "angustiada". Também não sentia mais "vontade de comer" e, desde a morte do companheiro, não fazia uma refeição completa, limitando-se à ingestão de amendoins e, ocasionalmente, "algum biscoito que ainda estivesse no armário".

Com as cobranças dos amigos para vê-la "sair dessa", afastou-se dos locais de convivência anteriormente frequentados. Evitava telefonemas e diz que, quando a procuravam, "arrumava uma desculpa para não ter que ver ninguém".

Acrescenta que, às vezes, ouvia algo que pensava ser o marido chamando-a pelo nome, e isso a deixava "tensa" e "muito triste", pois a fazia lembrar que não mais o veria. Conta que gostaria de não pensar tanto nisso, mas que não possui controle sobre esse fluxo de pensamentos.

Fisicamente, relata "dores de cabeça" constantes e dificuldades para dormir. Náuseas são associadas aos momentos em que pensa na morte do marido e fica imaginando "como deve ter sido na hora". Grande tristeza que, segundo ela, "chega ao desespero".

Diz que pensa várias vezes em se matar e que essa seria uma forma de estar próxima ao marido. Acrescenta que "no início era pior" e que agora "está melhorando", mas que "às vezes ainda pensa".

Pensa, ainda, em abandonar definitivamente o emprego, mas não o faz, pois tem que "continuar pagando as contas". Quanto às possibilidades de relacionamentos futuros, diz que "seria uma traição" ao marido e acredita que jamais terá outro relacionamento "desse tipo".

Por causa das queixas físicas, por insistência da mãe, única pessoa com quem ainda mantinha contato, procurou um médico clínico geral, que fez o encaminhamento da paciente para atendimento psicológico. Foi informado no encaminhamento que a paciente apresentava insônia e cefaleia, sem qualquer justificativa orgânica para os problemas relatados.

\section{Métodos}

Após avaliação da paciente, composta por entrevista e testagem psicológica, foi iniciado o processo terapêutico, que consistiu em 12 atendimentos clínicos, tendo duração de 45 minutos cada e com espaçamento de uma semana entre eles. Foi utilizado como base um protocolo de atendimento para luto ${ }^{12}$ no enfoque da terapia cognitivo-comportamental.

O protocolo, composto por técnicas tanto cognitivistas quanto comportamentais, tem como objetivo a psicoeducação do paciente em relação ao processo de enlutamento, a redução dos sinais e sintomas típicos do luto, a instrumentalização da paciente para a reformulação de papéis, considerando a necessidade de readaptação à vida, e o investimento em novos projetos futuros.

Didaticamente, o protocolo pode ser dividido em três meses. No primeiro mês, são fornecidos esclarecimentos quanto ao processo de luto considerando suas diferentes fases (Alarme/Torpor/Procura/ Depressão/Reorganização) ${ }^{5}$. O paciente aprende a identificar sinais e sintomas, assim como técnicas para controle da ansiedade e da depressão em momentos agudos. Recebe treinamento em mudança de foco. É enfatizado o reconhecimento da realidade da perda, assim como o compartilhamento dessa experiência. Há elaboração de ritual de despedida respeitando as crenças e a cultura do paciente.

No segundo mês, são trabalhadas questões como a resolução de problemas pendentes entre o sujeito enlutado e o ser perdido, criação de uma rede social de apoio, reorganização dentro do sistema familiar e redistribuição de papéis. O terceiro mês tem o objetivo de propiciar a readaptação da pessoa à vida cotidiana, organização dos horários de atividades semanais, considerando a ausência do ser perdido, facilitação do investimento em novos objetivos de vida e novas relações, além da prevenção de recaída.

O progresso da paciente foi acompanhado ao longo das 12 semanas com os seguintes instrumentos: Inventário Beck de Depressão (BDI), Inventário Beck de Ansiedade (BAI) e Escala Beck de Desesperança (BHS) - escalas de autorrelato que medem, respectivamente, intensidade de depressão, ansiedade e desesperança -, Inventário de Sintomas de Stress de Lipp (ISSL) - instrumento para avaliar estresse segundo o modelo quadrifásico - e Questionário de Saúde Geral (QSG).

O QSG busca quantificar a gravidade de distúrbios psiquiátricos menores e trabalha com cinco fatores: 1) estresse psíquico; 2) desejo de morte; 3) desconfiança no próprio desempenho; 4) distúrbios do sono e 5) distúrbios psicossomáticos. A interpretação dos dados coletados com todos os instrumentos utilizados foi realizada com base nos manuais ${ }^{13-15}$ e considerando normas estabelecidas para a população brasileira.

A participante da pesquisa teve assegurado seu direito à privacidade e foi informada sobre objetivos e procedimentos do estudo, assim como sobre possíveis formas de utilização dos dados coletados. A pesquisa é aprovada por comitê de ética em pesquisa e a participante assinou Termo de Consentimento Livre e Esclarecido.

\section{Resultados}

Na tabela 1 são apresentados os resultados das aplicações de BDI, BAI, BHS e ISSL e os resultados dos cinco fatores do QSG (em percentil), considerando-se início e final do tratamento.

Tabela 1. Resultados: BDI, BAI, BHS, ISSL (classificação) e OSG (percentil para cada um dos cinco fatores)

\begin{tabular}{l|c|c}
\hline Semanas & 1 & 12 \\
\hline BDI & $56(G)$ & $32(\mathrm{M})$ \\
\hline BAI & $47(G)$ & $21(\mathrm{M})$ \\
\hline BHS & $19(\mathrm{G})$ & $06(\mathrm{~L})$ \\
\hline ISSL & $\mathrm{E}$ & $\mathrm{R}$ \\
\hline OSG -1 & 95 & 50 \\
\hline OSG -2 & 95 & 20 \\
\hline OSG -3 & 90 & 40 \\
\hline OSG -4 & 95 & 45 \\
\hline OSG -5 & 95 & 60 \\
\hline
\end{tabular}

Abreviações: G: grave; M: moderado; L: leve; BDI: Inventário Beck de Depressão; BAl: Inventário Beck de Ansiedade; BHS: Escala Beck de Desesperança; ISSL: Inventário de Sintomas de Stress de Lipp; E: exaustão; R: resistência; QSG-1: estresse psíquico; OSG-2: desejo de morte; QSG-3: desconfiança no próprio desempenho; QSG-4: distúrbios do sono; QSG-5: distúrbios psicossomáticos.

Houve redução em todos os itens mensurados. A depressão passou de grave (56) para moderada (32), e a ansiedade, também grave (47) inicialmente, foi reduzida para moderada (21), estando bem próxima a leve (ponto de corte: 19/20). A desesperança passou de grave (19) para leve (6). Em relação ao estresse, a paciente estava em exaustão quando iniciou o tratamento e em resistência ao final, mostrando progresso.

Houve redução do estresse psíquico (de 95\% para 50\%), da desconfiança em relação ao próprio desempenho (de $90 \%$ para $40 \%$ ), de 
distúrbios do sono (de $95 \%$ para $45 \%$ ) e também de distúrbios psicossomáticos (de $95 \%$ para $60 \%$ ) e do desejo de morte (de $95 \%$ para $20 \%$ ).

Ao final das 12 semanas, a paciente já se encontrava frequentando normalmente $\mathrm{o}$ ambiente de trabalho, tinha retomado o contato com amigos (inicialmente por telefone e, posteriormente, encontrando-os pessoalmente) e, apesar de relatar não desejar nenhum novo relacionamento afetivo no momento, considerava essa possibilidade para o futuro como algo positivo em sua vida.

\section{Conclusão}

Estudos voltados para terapêutica do luto ainda são escassos na literatura, sendo ausentes estudos sobre a eficácia da terapia cognitivo-comportamental para luto, considerando as particularidades enfrentadas por aqueles que sofrem com a perda súbita de seus cônjuges.

Por meio do atendimento com enfoque cognitivista-comportamental, foi possível a obtenção de melhora em todas as medidas de avaliação realizadas: depressão, ansiedade, esperança em relação ao futuro, estresse, habilidades sociais, distúrbios do sono e distúrbios psicossomáticos. O acompanhamento do progresso obtido por meio de instrumentos psicométricos validados, com boa fidedignidade e com normas estabelecidas para a população brasileira garante a qualidade da avaliação realizada.

O período mais propício para prevenção de episódios depressivos após o luto é aquele próximo à ocorrência da morte7; desse modo, o fato de a paciente ter iniciado o acompanhamento terapêutico dois meses após a perda pode ser sido benéfico. São necessários, no entanto, novos estudos para avaliar a real influência do período transcorrido após a perda na eficácia terapêutica, não apenas em relação à profilaxia de episódios depressivos, mas também em relação a outros sinais e sintomas do luto.

O estudo de caso apresentado colabora para a ampliação dos limites da terapia cognitivo-comportamental, apresentando bons resultados para o tratamento específico de um quadro de luto decorrente da morte súbita de cônjuge.

\section{Nome da agência de fomento que concedeu auxílio}

Conselho Nacional de Desenvolvimento Científico e Tecnológico (CNPq).

\section{Declaração de conflito de interesses}

Os autores declaram que não há conflitos de interesse relativos a este estudo.

\section{Referências}

1. Zisook S, Shear K. Grief and bereavement: what psychiatrists need to know. World Psychiatry. 2009;8:67-74.

2. Hensley PL, Clayton PJ. Bereavement: signs, symptoms, and course. Psychiatr Ann. 2008;38:649-54.

3. Karam EG, Tabet CC, Alam D, Shamseddeen W, Chatila Y, Mneimneh $Z$, et al. Bereavement related and non bereavement related depressions: a comparative field study. J Affect Disord. 2009;112:102-10.

4. Clayton PJ. Bereavement and depression. J Clin Psychiatry. 1990;51:34-8.

5. 5. Parkes CM. Luto: estudos sobre a perda na vida adulta. São Paulo: Summus; 1998.

6. Stroebe W, Abakoumkin G, Stroebe M. Beyond depression: yearning for the loss of a loved one. Omega (Westport). 2010;61:85-101.

7. Brent D, Melhem N, Donohoe MB, Walker M. The incidence and course of depression in bereaved youth 21 months after the loss of a parent to suicide, accident, or sudden natural death. Am J Psychiatry. 2009;166:786-94.

8. Rodger ML, Sherwood P, O'Connor M, Leslie G. Living beyond the unanticipated sudden death of a partner: a phenomenological study. Omega (Westport). 2006-2007;54:107-33.

9. Clayton PJ. Bereavement and depression. J Clin Psychiatry. 1990;51:34-8.

10. Stroebe M, Schut H, Stroebe W. Health outcomes of bereavement. Lancet. 2007;370:1960-73.

11. Prigerson HG, Horowitz MJ, Jacobs SC, Parkes CM, Asian M, Goodkin K, et. al. Prolonged grief disorder: psychometric validation of criteria proposed for DSM-V and ICD-11. Plos Med. 2009;6:1-12.

12. Silva ACO. Atendimento clínico para luto no enfoque da terapia cognitivo-comportamental. In: Wielenska RC (Org.). Sobre comportamento e cognição: desafios, soluções e questionamentos. Santo André: ESETec; 2009.

13. Cunha JC. Manual da versão em português das escalas Beck. São Paulo: Casa do Psicólogo; 2001.

14. Lipp MN. Manual do Inventário de Sintomas de Stress para Adultos de Lipp (ISSL). São Paulo: Casa do Psicólogo; 2000.

15. Goldberg DP. Questionário de Saúde Geral: manual técnico. São Paulo: Casa do Psicólogo; 1996 